\title{
EL TESTAMENTO DEL ESCULTOR GREGORIO HERNÁNDEZ (SEVILLA, 1615)
}

\author{
THE SCULPTOR GREGORIO HERNÁNDEZ'S \\ TESTAMENT (SEVILLE, 1615)
}

\author{
Francisco Manuel Delgado Aboza \\ Colegio Cristo Rey, Sevilla. España \\ fdelgado@cristoreysevilla.es
}

\begin{abstract}
La escasa documentación sobre el escultor Gregorio Hernández, del que únicamente tenemos datos de algunos de sus trabajos, le proporciona mayor interés al hallazgo de su testamento en el Archivo Histórico Provincial de Sevilla. Dicha escritura, que se fecha en 1615, nos aporta diversas noticias inéditas de su vida, lo que permitirá conocer algo mejor la figura de este artista.

Palabras claves: Gregorio Hernández; escultor; testamento; Sevilla; siglo XVII.
\end{abstract}

The scant documentation about the sculptor Gregorio Hernández, of which we only have data of some of his works, provides greater interest to the discovery of his testament at the Seville Provincial Historical Archive. This writing, dated in 1615, brings us various unpublished news about his life, which will allow us to know better the figure of this artist.

Keywords: Gregorio Hernández; sculptor; testament; Seville; $17^{\text {th }}$ century.

Dentro de la rica y notable nómina de escultores que vivieron en la Sevilla de las últimas décadas del siglo XVI y primeros años del siguiente ${ }^{1}$, atraídos por la importancia de la bulliciosa y cosmopolita metrópoli hispalense, pilar sobre el que se cimentó una parte destacada de la producción artística de nuestro país, además de los maestros más conocidos, encontramos otros autores que

${ }^{1}$ RODA PEÑA, José: "La escultura sevillana a finales del Renacimiento y en los umbrales del Naturalismo", en GILA MEDINA, Lázaro (coord.): La escultura del primer Naturalismo en Andalucía e Hispanoamérica (1580-1625). Madrid, 2010, pp. 273-306; y "El triunfo del naturalismo en la escultura sevillana y su introducción al pleno barroco", en GILA MEDINA, Lázaro (coord.): La consolidación del Barroco en la escultura andaluza e hispanoamericana. Granada, 2013, pp. 143-178. 
por distintas circunstancias han pasado más desapercibidos para los historiadores. Uno de ellos, sin duda, es el maestro escultor Gregorio Hernández, del que sabemos muy pocos datos, aunque como veremos trabajó en dos de los más afamados edificios de Sevilla; nos referimos al Alcázar y a la antigua Casa Lonja, actualmente Archivo General de Indias.

Es el escritor e historiador José Gestoso el primero que recoge la existencia de este artista, antes en su Sevilla Monumental y Artística y luego en el conocido Diccionario de artífices sevillanos entre los siglos XIII y XVIII, catalogándolo como entallador. En su escueto comentario nos dice que participó en los trabajos que se efectuaron en el Alcázar desde el año 1596, "en cuya Hijuela comienza á citársele" ${ }^{2}$. No obstante, apuntamos que la referencia documental más antigua de su presencia en el Real Alcázar se fecha algunos años antes, en concreto en 1593, cuando aparece como entallador trabajando en el alfarje del corredor del Jardín del Príncipe, lo que continuaba haciendo en 1594, junto a otras reparaciones de menor importancia. Entre 1595 y 1598 está ocupado, sobre todo, en las techumbres de las salas colaterales a la media naranja o Salón de Embajadores. Años más tarde, desde abril de 1610, localizamos nuevas referencias a Gregorio Hernández, esta vez como escultor, al que se expide en mayo la cantidad de 56 reales por dos cabezas y cuatro manos de madera "que hizo para las figuras del jardin", posiblemente el de las Damas ${ }^{3}$.

Para la Lonja de Mercaderes realiza en 1611 la talla de la bóveda que cubre la escalera situada en la crujía norte del edificio y que comunica con sus azoteas, cuyo diseño se debe a Miguel de Zumárraga (Figura 1). Dicha bóveda destaca por su decoración concéntrica de escamas y por la gran cartela central con el anagrama "IHS" entre dos cabezas aladas de querubines. A partir del citado año 1611, Zumárraga realiza otro significativo cambio que afectará al exterior de la Lonja, que consistió en ampliar el número de puertas en cada frente, de una a tres, labrándose por Gregorio Hernández las cartelas apaisadas que se ubican bajo el dintel de estas nuevas entradas (Figura 2). Al año siguiente realizó la urna o basamento de la Cruz del Juramento situada delante de la fachada norte de la Lonja -cuya posición central actual data de 1760-, habiendo sido policromada y dorada por Amaro Vázquez, que hizo lo propio con la reja que la protege (Figura 3). Parece que estas labores coincidieron con la terminación de las bóvedas de las naves superiores del frente norte, cuya decoración la llevó a cabo Gregorio Hernández,

${ }^{2}$ GESTOSO Y PÉREZ, José: Sevilla Monumental y Artística. T. I. Sevilla, 1889, pp. 642-644; y Ensayo de un Diccionario de los artífices que florecieron en Sevilla desde el siglo XIII al XVIII inclusive. T. I. Sevilla, 1899, p. 187.

3 MARÍN FIDAlGO, Ana: El Alcázar de Sevilla bajo los Austrias. T. II. Sevilla, 1990, pp. 392, 724 y 784. 
que continuaba trabajando al año siguiente en el ornato de las bóvedas de los costados sur y este, además de reparar la fuente que había en el centro del patio 4 .

Aparte de las obras anteriormente señaladas, apuntamos cómo en el conocido manuscrito La Charidad Guzmana, escrito por el dominico fray Pedro Beltrán en 1612 y en el que se hace un encendido enaltecimiento de las riquezas de Sanlúcar de Barrameda, se menciona en su canto IV a un escultor, llamado Gregorio Hernández y afincado en Sevilla, como el autor de la Virgen de la Caridad, patrona de esta localidad gaditana; asimismo, también se habla de un pintor apellidado Bracamonte como el artífice de su policromía. Este último artista se viene identificando con el pintor y dorador Baltasar López de Bracamonte, del que sabemos que trabajó en el Alcázar de Sevilla entre los años 1590 y 1591, lo que pudo significar que coincidiera -según apunta el profesor Fernando Cruz Isidoro- con el escultor Gregorio Hernández .

Centrándonos en el testamento de Gregorio Hernández, vemos que sigue uno de los modelos más habituales y empleados en este género de documentos, el llamado testamento abierto. Este se otorga en presencia del escribano y de los testigos, manifestándose por el otorgante las cláusulas o condiciones y designándose sus herederos. El documento que nos ocupa, que se fecha en Sevilla el 4 de junio de 1615 ante el escribano público Antonio de Medina Sánchez, aparece firmado por el citado notario, el propio artista y, como testigos, los escribanos de Sevilla Diego Jiménez, Gonzalo Morán y Diego de Zuloaga. Al comienzo de la escritura se informa que Gregorio Hernández, que se identifica como maestro escultor, era vecino de la ciudad de Sevilla, en la collación de San Martín. Seguidamente, se aclara que estaba "enffermo del cuerpo e sano de la voluntad y en todo mi acuerdo juiçio y entendimiento natural y en mi cumplida e buena memoria tal qual Dios nuestro señor fue seruido de me dar creyendo como creo en todo lo que tiene e cree la Santa madre yglesia de Roma otorgo y hago mi testamento en la forma e manera siguiente" 6 .

La primera cláusula testamentaria establece el lugar de su enterramiento, que no sería otro que la parroquia de donde era feligrés, la de San Martín, "en la

${ }^{4}$ MORALES, Alfredo J.: "Lonja de Mercaderes y Archivo General de Indias", en Archivo General de Indias. Barcelona, 1995, pp. 60-61; y "El Archivo de Indias", Apuntes del Real Alcázar de Sevilla, 14, 2013, pp. 50-71. Véase también PLEGUEZUELO HERNÁNDEZ, Alfonso: "La Lonja de Mercaderes de Sevilla: de los proyectos a la ejecución", Archivo Español de Arte, 249, 1990, pp. 15-41.

5 CRUZ ISIDORO, Fernando: El Santuario de Ntra. Sra. de la Caridad, de Sanlúcar de Barrameda. Estudio Histórico-Artístico. Córdoba, 1997, pp. 21-26 y 249-252; y El patrimonio restaurado de la Basílica de la Caridad de Sanlúcar de Barrameda. Sanlúcar de Barrameda, 2011, pp. 27-38.

${ }^{6}$ Archivo Histórico Provincial de Sevilla, sección Protocolos Notariales de Sevilla, leg. 341, of. 1, 1615, ff. 934v-935v. 
sepultura que me fuere dada". En este punto hay que aclarar que entre la documentación histórica del citado templo, que actualmente forma parte del archivo parroquial de San Andrés, encontramos un salto cronológico en los libros de defunciones, pasando del volumen registrado con el número 1, que abarca los años 1589 a 1591, al libro $2^{\circ}$, que comienza el 17 de febrero de 1617 y concluye el 3 de diciembre de $1636^{7}$. Lamentablemente, esto nos impide verificar si finalmente se cumplió su deseo de ser inhumado en esta iglesia y la fecha exacta, aunque sí podemos anotar que en el aludido libro $2^{\circ}$ no hay ninguna referencia al citado escultor, lo que nos permite especular que se realizara con anterioridad. Por otro lado, se pide que en el día de su entierro, si daba lugar -"siendo hora"-, o en el día que se pudiera, se dijeran por su alma una misa de réquiem cantada y cuatro misas, además de llevarse la ofrenda ${ }^{8}$; asimismo, se aclara que todos los pormenores del sepelio se harían siguiendo los dictámenes de sus albaceas.

Además de estas misas, el escultor manda se oficiasen otras tres a la Santísima Trinidad, "en altar donde se saca anima"; aunque no se especifica creemos que se refiere a la propia iglesia de San Martín, al altar de las Ánimas que existía en el muro del evangelio, cuyo retablo y pintura fueron renovados a finales del XVIII"; una misa rezada a San Miguel y otras dos a "nuestra Señora del Rosario", estas tres últimas en donde quisieran sus albaceas. De la misma forma, ordena que se dijeran por él "las demas missas officios e sacrificios" que tuviera a bien su mujer Francisca Márquez, dejando bien claro "que ningun juez ni prelado le pida quenta si las a dicho o no porque respecto de no tener como al presente no tengo bienes de que se pueda cumplir este testamento lo dejo a voluntad de la dicha mi mujer si quisiere haserme decir mas missas o no".

Siguiendo con las cláusulas del testamento, y como era común en esa época, se fija una donación de cuatro reales para la cera del Santísimo Sacramento y otros tantos para la de las Ánimas del Purgatorio, ambas corporaciones de la parroquia de San Martín" . Asimismo, establece que se donara a las conocidas "mandas acostumbradas", también llamadas "forzosas", práctica muy habitual en la mayoría de los testamentos de aquella época, la cantidad de ocho maravedíes a cada parte. Esta

${ }^{7}$ MORALES PADRÓN, Francisco: Los archivos parroquiales de Sevilla. Sevilla, 1982, p. 297.

${ }^{8}$ Estas ofrendas en los funerales, que estaban muy enraizadas en el pueblo, se convirtieron en un modo de diferenciación social, según el valor y cuantía de las mismas. DOMÍNGUEZ ORTIZ, Antonio: La sociedad española en el siglo XVII. T. II. Granada, 1992, pp. 56-57.

9 ESCUDERO MARCHANTE, José María: La iglesia de San Martín de Tours de Sevilla. Historia y Descripción Artística. Sevilla, 2007, p. 20.

${ }^{10}$ Algunos años más tarde, en 1632, se verificó la unión de ambas hermandades. ESCUDERO MARCHANTE, José María: "La fusión de la Hermandad Sacramental de San Martín con la de Ánimas Benditas: año 1632”, Boletín de las Cofradías de Sevilla, 477, 1998, pp. 38-39. 
costumbre se hizo tan popular que no se consideró imprescindible su enumeración en los testamentos, como vemos en el de Gregorio Hernández. Los beneficiarios más habituales eran los Santos Lugares de Jerusalén, la redención de cautivos o los niños expósitos.

En el siguiente punto el escultor declara que disfrutaba de "unas casas en que al presente biuo que son en la dicha collacion de San Martin en la calle que dizen de la mata", aunque la propiedad correspondía a la fábrica parroquial de San Andrés, a la que se pagaba una renta anual -nada despreciable- de 10.000 maravedíes. Dicha calle de la Mata, situada muy cerca de la Alameda de Hércules, se constituye en dos espacios: el comienzo se conocía con la denominación referida desde el primer tercio del siglo XVII, mientras que el tramo final se llamó plaza de Belén, por el convento del mismo nombre allí ubicado. Tras la supresión de este cenobio carmelita, con el traslado en 1837 de sus monjas al convento de Santa Ana ${ }^{11}$, se unificó todo el lugar con el topónimo actual ${ }^{12}$. Asimismo, se aclara que Gregorio Hernández tenía la facultad de nombrar a un heredero para la conocida como "segunda vida de las dichas casas", señalándose como tal a su esposa Francisca, "para que desde el dia de mi fallecimiento en adelante durante los dias de su bida goze de las dichas cassas y pague la renta del señorio e cumpla las condiciones que yo estoy obligado por la escritura de arrendamiento".

Por último, para asegurarse que se cumpliera con todo lo establecido en su testamento y con las mandas y cláusulas del mismo, señalaba a su mujer como su albacea, a quien le daba poder cumplido "para que use e pueda usar del cargo de albaçea" y vender de sus bienes los que necesitara para la observancia del testamento. Del mismo modo, una vez verificado con todo lo que disponía el documento, dejaba y nombraba "por mi heredero unibersal en todos mis bienes derechos e aciones a la dicha Francisca marques mi mujer para que como tal mi heredera aya para si los dichos mis bienes derechos e aciones por no tener como no tengo heredero forzoso que herede los dichos mis bienes". Finalmente, anulaba todos los testamentos, mandas, codicilos y otras disposiciones anteriores a la presente escritura testamentaria.

Fecha de recepción: 30 de septiembre de 2016

Fecha de aceptación: 4 de enero de 2017

${ }^{11}$ FRAGA IRIBARNE, María Luisa: Conventos femeninos desaparecidos. Sevilla. Siglo XIX. Sevilla, 1993, p. 79.

12 COLLANTES DE TERÁN SÁNCHEZ, Antonio: "MATA, calle de la", en Diccionario histórico de las calles de Sevilla. T. II. Sevilla, 1993, p. 84. 


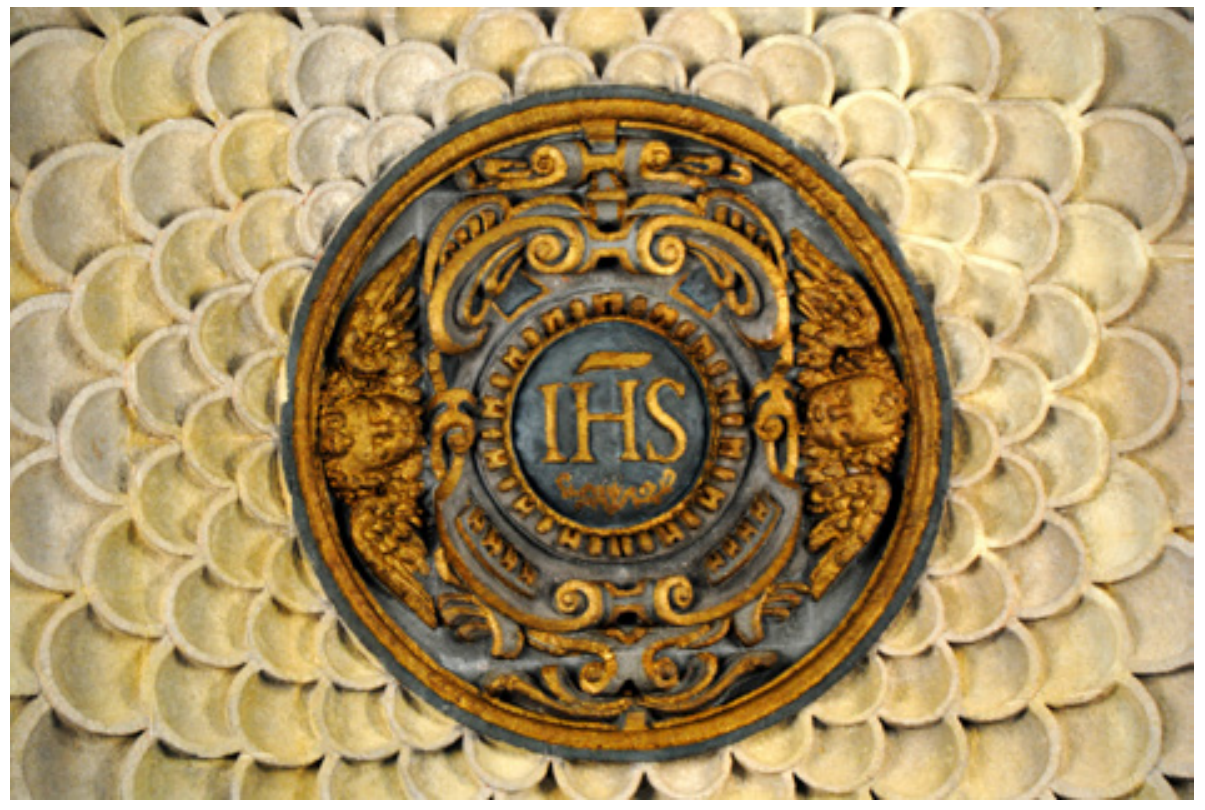

Figura 1. Gregorio Hernández, Bóveda de escalera (detalle), Archivo General de Indias, Sevilla. 


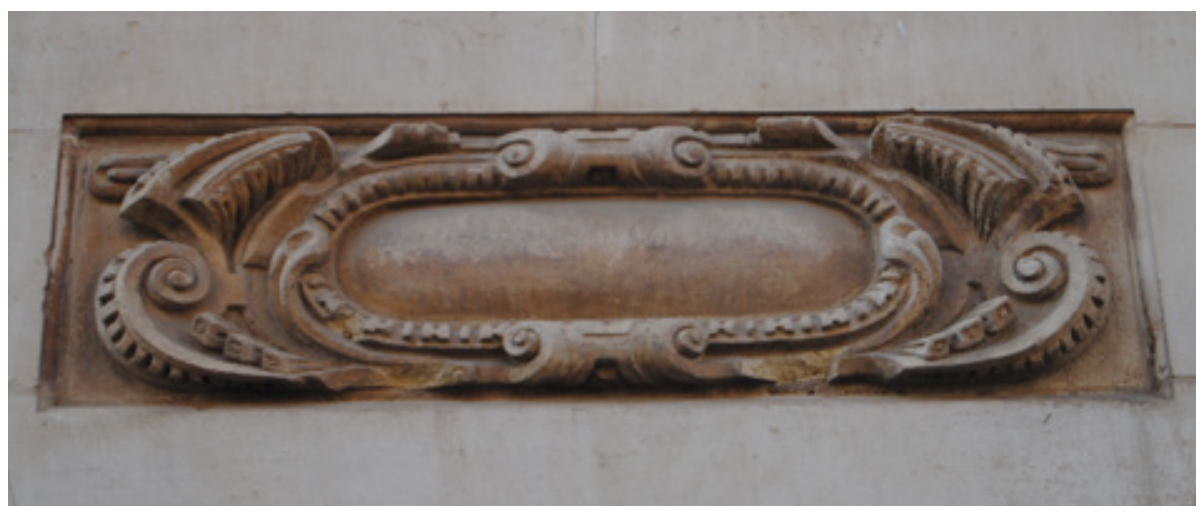

Figura 2. Gregorio Hernández, Cartelas apaisadas, Archivo General de Indias, Sevilla. 


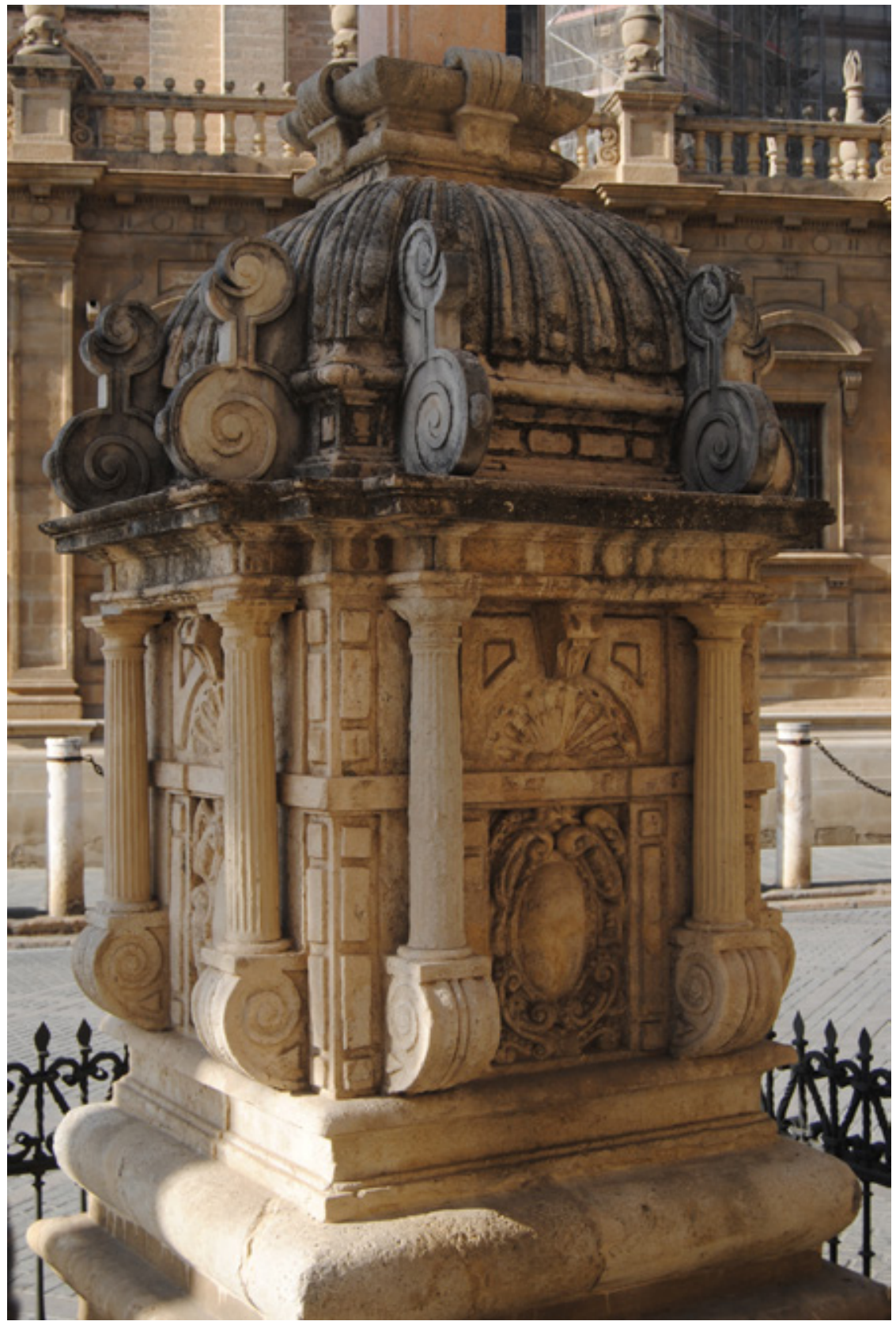

Figura 3. Gregorio Hernández, Basamento de la Cruz del Juramento, Archivo General de Indias, Sevilla.

LABORATORIO DE ARTE 29 (2017), pp. 795-802, ISSN 1130-5762

e-ISSN 2253-8305 - DOI http://dx.doi.org/10.12795/LA.2017.i29.43 